\title{
ORIGINAL
}

\section{Resistance to high-fat diet-induced obesity in male heterozygous Pprc1 knockout mice}

\author{
Nan $\mathrm{Zhai}^{1)^{*}}$, Chen Sun ${ }^{1), 2)^{*}}$, Weiqiong $\mathrm{Gu}^{1)^{*}}$, Xin $\mathrm{He}^{1), 2)}$, Aijing Shan ${ }^{1)}$, Hanxiao Sun ${ }^{1)}$, Nan $\mathrm{Lu}^{1)}$, \\ Bin Cui ${ }^{1), 2)}$ and Guang Ning ${ }^{1), 2)}$ \\ 1) Shanghai Clinical Center for Endocrine and Metabolic Diseases, Shanghai Institute of Endocrinology and Metabolism, \\ Endocrine and Metabolic E-Institutes of Shanghai Universities and Key Laboratory for Endocrinology and Metabolism of \\ Chinese Health Ministry, Rui-jin Hospital, Shanghai Jiao-Tong University School of Medicine, Shang Hai 200025, China \\ 2) Laboratory of Endocrinology and Metabolism, Institute of Health Sciences, Shanghai Institutes for Biological Sciences, Chinese \\ Academy of Sciences, and Shanghai Jiao-Tong University School of Medicine, Shang Hai 200025, China
}

\begin{abstract}
Peroxisome proliferator-activated receptor gamma, co-activator-related 1 (Pprc1) is the third member of the Pgc1 family. Other than the well-characterized Pgcla and Pgclb that act as regulators of mitochondrial biogenesis and oxidative metabolism, the function of Pprc1 in vivo is rarely reported, due to embryonic lethality of whole-body Pprc1 knockout mice. To investigate the biological and physiological function of Pprc1 in metabolic processes, male $\mathrm{Pprcl}^{+/-}$ mice fed with a high fat diet (HFD) showed resistance to diet-induced obesity with a decrease of adipose tissue in $\mathrm{Pprcl}^{+/-}$ mice, which was a result of elevated energy expenditure. In skeletal muscle of $P p r c 1^{+/}$mice, Pprc1 level showed haploinsufficiency with down-regulation of Pgc1b and Pgcla, whereas in adipose tissue, Pprc1 expression remained normal, with significant compensatory increase of other Pgc1 family members to induce an up-regulation of respiratory chain genes. Taken together, as the first report on the metabolic roles of Pprc1 in vivo, these results indicated an elevated basal metabolic rate and lipid metabolic alteration of male $\mathrm{Pprcl}^{+/}$mice on HFD, suggesting the significant role of Pprc1 in controlling mitochondrial gene expression and energy metabolic processes, synergistically with Pgcla and Pgclb.
\end{abstract}

Keywords: Pprc1, Pgc1 family, High-fat diet, Obesity, Energy metabolism

WORLDWIDE prevalence of obesity and related metabolic diseases highlights the problem of energy balance and energy metabolism. Environmental factors, such as high-fat diets (HFDs), high caloric intake, and sedentary lifestyles, along with genetic variations, contribute to the pathogenesis of obesity. Pgcla and Pgc1b potently induce the expression of a series of genes involved in energy homeostasis, and act as pleiotropic regulators of mitochondrial biogenesis and oxidative metabolism [1-4]. Pgcla ${ }^{-/-}$mice were protected from diet-induced obesity $[5,6]$, whereas transgenic mice of Pgcla were severely obese [7]. In contrast, both transgenic and knockout of Pgclb in mice were resistant to diet-induced obesity [8-10]. Meanwhile,

Submitted Aug. 19, 2014; Accepted Apr. 9, 2015 as EJ14-0383 Released online in J-STAGE as advance publication May 20, 2015 Correspondence to: Bin Cui, Ph.D., Bldg. 1, Room 106, Laboratory of Endocrinology and Metabolism, Institute of Health Sciences, Shanghai Institutes for Biological Sciences, Chinese Academy of Sciences, and Shanghai Jiao-Tong University School of Medicine, 225 South Chongqing Road, Shang Hai 200025, China.

E-mail: bcui@sibs.ac.cn

* These authors contribute equally to the work.

(C)The Japan Endocrine Society accumulating evidence associated genetic variations of these two members of Pgc1 family with human obesity and related diseases [2, 4, 11-16].

In vitro studies demonstrated that Pprc1, the third member of the Pgc1 family, interacts with transcription factors including Nrf1, Nrf2, Erra, Creb (cAMPresponse element-binding protein), etc., and thus contributes to mitochondrial biogenesis and orchestrates response to metabolic stress by promoting the expression of multiple genes specifying inflammation, proliferation, and metabolic reprogramming [17-21]. Moreover, a human genetic analysis on type 2 diabetes showed a marginal significant association $(\mathrm{OR}=1.06$ [95\% CI: 1.00-1.13], $P=0.06$ ) between PPRC1 and type II diabetes, which suggested function of PPRC1 in human metabolic diseases [17]. Whole-body knockout of Pprc1 in mice causes embryonic lethality [18], hence reports on physiological function of the gene in vivo are quite rare. As the first report on metabolic roles of Pprc1 in vivo, the present study demonstrates that Pprc1+/- mice are protected from HFD-induced obesity with a prominent decrease of adipose tissue as a 
result of elevated energy expenditure.

\section{Materials and Methods}

\section{Mice}

Pprcl $^{+/-}$mice were generated by homologous recombination as previously described [18]. Fiveweek-old male $\mathrm{PprCl}^{+/+}$and $\mathrm{Pprcl}^{+/-}$littermate mice were randomized to either high-fat (HFD, D12492, Research Diets, New Brunswick, NJ, USA) or normal chow diet (ND) ad libitum for 13 weeks. Mice were housed individually for four consecutive days to compare food consumption and body temperature.

Experiments were performed with permission and followed the recommendations for the protection of Vertebrate Animals used for Experimental and Scientific purposes of the local Animal Care Committee in Shanghai Jiao-Tong University School of Medicine.

\section{Metabolic tests of glucose homeostasis}

Mice were fasted overnight prior to intra-peritoneal glucose tolerance test (IPGTT, $2 \mathrm{~g}$ glucose $/ \mathrm{kg}$ body weight) and pyruvate tolerance test (PTT, 2 g sodium pyruvate $/ \mathrm{kg}$ ) or $8 \mathrm{hr}$ before insulin tolerance test (ITT, $0.75 \mathrm{U}$ insulin $/ \mathrm{kg}$ ). Blood glucose was monitored using OneTouch Ultra glucose meter (LifeScan, High Wycombe, Buckinghamshire, UK).

\section{Body composition and indirect calorimetry}

Nuclear magnetic resonance (NMR, Bruker Minispec, Hannover, Germany) was performed for body composition analysis. Oxygen consumption and carbon dioxide production were measured and correlated to individual body weights with Oxymax (Columbus Instruments International, Columbus, OH, USA), and then respiratory exchange ratio (RER) and heat were calculated accordingly [19].

\section{Biochemical analysis}

Enzymatic assay kits were used for determination of serum non-esterified fatty acid (NEFA, Wako, Osaka, Japan), triglyceride (Rsbio, Shanghai, China), cholesterol (Rsbio), and aspartate transaminase (AST, Rsbio) according to the manufacturer's instructions.

\section{Real-time PCR}

Total RNA was extracted from tissues of 22-weekold mice using Trizol Reagent (Invitrogen), and then reverse-transcribed with Reverse Transcription System
(Promega, Madison, WI, USA). Total DNA was extracted using DNeasy Blood \& Tissue Kit (Qiagen). Real-time PCR was performed with Sybr-Green (Takara, Shiga, Japan) on LightCycler 480 (Roche, Basel, Switzerland) using GADPH for RNA and 18s RNA for mitochondrial DNA as internal reference. Primer sequences used are listed in Table S1.

\section{Western blot}

Brown adipocytes were lysed with $1 \%$ sodium deoxycholate, $1 \%$ Triton $\mathrm{X}-100$ and protease inhibitor cocktail (Roche Applied Science). After $10 \mathrm{~min}$ of microcentrifugation at $20,000 \mathrm{x}$ in $4^{\circ} \mathrm{C}$, the supernatant liquid was separated by SDS-PAGE, and then transferred to nitrocellulose membranes. Antigens were visualized by sequential treatment with specific antibodies. Goat anti-rabbit or anti-mouse IgG (1:4000; ZSGB-BIO Inc., Beijing, China) were used as the secondary antibody. Immunohistochemistry immune complexes were detected by using Immobilon Western Chemiluminescent HRP Substrate kit (Merck Millipore, Darmstadt,Germany).

\section{Statistical analysis}

Data are expressed as mean \pm SEM. Statistical analyses were performed with Student's $t$ test for independent samples. For growth curve analysis, one-way repeated-measures ANOVA and Bonferroni multiple comparisons test were performed. ${ }^{*} p<0.05 ;{ }^{* *} p<$ $0.01 ; * * * p 0.001$.

\section{Results}

Growth curves and body composition in $\mathrm{Pprcl}^{+/-}$mice

Given the fact that homozygous mutation of Pprcl causes embryonic lethality, $\mathrm{Pprcl}^{+/}$mice were generated from homologous recombination as previously reported [18]. When fed on ND, $\mathrm{PprCl}^{+/-}$mice appeared normal, with body weights similar to wildtype (WT) littermates (Fig. 1a). However, Pprcl ${ }^{+/-}$ mice showed significantly less weight gain than WT on HFD (Fig. 1b), which was consistent with fat content reduction $(-15.3 \%, p=0.0244)$ of $\mathrm{Pprcl}^{+/}$mice (Fig. 1c). Meanwhile, the relative lean mass of $\mathrm{Pprcl}^{+/-}$ mice increased significantly $(+19.0 \%, p=0.0080$ in male; $+39.4 \%$, ) compared to WT (Fig. 1d).

\section{Glucose homeostasis and serum chemistry in Pprc1 ${ }^{+/}$mice} Body mass reduction may occur as a result of glu- 
cose homeostasis disorder. IPGTT, ITT and PTT were conducted in $\mathrm{Pprcl}^{+/}$and WT mice on both ND and HFD, but showed no differences (Fig. S1a-c). Serum chemistry profiles further revealed that $\mathrm{Pprcl}^{+/-}$mice had almost identical T-C, HDL-C, LDL-C, NEFA, and AST levels with WT, and yet TG level showed a $105.7 \%$ increase $(p=0.0001)$ (Fig. S1d-g).

Elevated energy consumption in $\mathrm{Pprcl}^{+/-}$mice on HFD

Unbalanced energy intake and expenditure contribute to obesity and leanness. However, $\mathrm{PprCl}^{+/-}$mice had comparable food consumption (Fig. 1k) and body temperature (Fig. 1j) with WT on HFD. Subsequently, energy expenditure of the mice was studied with indirect calorimetry. Increased $\mathrm{O}_{2}$ consumption (Fig. 1e) and $\mathrm{CO}_{2}$ production (Fig. 1f) both in daylight and in dark and higher heat consumption (Fig. 1i) were evident, indicating significantly increased energy expenditure in $\mathrm{PprCl}^{+-}$mice on HFD. In addition, respiratory exchange ratio (RER) of $\mathrm{Pprcl}^{+/}$mice decreased (from 0.789 to $0.782, p=0.0062$ ) in light cycle (Fig. 1i-j), with unaltered locomotor activity (Fig. 1h), suggesting an increase of fat oxidation in $\mathrm{Pprcl}^{+/}$mice on HFD.

\section{Decreased adipose tissue and mitochondrial content alternation in Pprc $^{+/-}$mice on HFD}

Dissection of fat deposits further showed that Pprc1+/- mice were protected from diet-induced obesity. Subcutaneous white adipose tissue (WAT) (subWAT; $-55.6 \%, p=0.0349$ ) (Fig. 2a) were significantly decreased in $\mathrm{PprCl}^{+/-}$mice. Interestingly, brown adipose tissue (BAT) was also decreased $(-22.2 \%, p=$ 0.0068) (Fig. 2b). Histology HE staining showed decreased lipid droplet diameter of Pprc1+/- mice both in BAT and subWAT (compared with their littermates (Fig. 2c). In BAT, expression analysis of mitochondrial content showed a 3.72-fold $(p=0.0013)$ increase in Pprcl $^{+/}$mice (Fig. 2o), consisted with the increased protein level of UCP1 (Fig. 2h.i). It suggested that BAT is a vital organ for the leanness phenotype of these mice.

\section{Altered expression profiles in Pprc1 ${ }^{+/}$mice on HFD}

Expression analysis of Pgc1 family genes (Fig. 2e-g) revealed that in Pprc1 highly expressed tissues [20], $\mathrm{PprCl}^{+-}$mice on HFD did not necessarily show an insufficient expression. Meanwhile, the compensatory increase of Pgclb (1.53-fold, $p=0.0256$ ) in WAT (Fig. 2e) and Pgcla (4.98-fold, $p=0.0254$ ) in BAT (Fig. 2f) was consistent with previously reported up-regulation of Pgcla in the absence of Pgclb [17, 21]. In skeletal muscle, the expression of Pprc1 decreased by $66.2 \%$ ( $p$ $=0.0402)$, along with a $57.9 \%$ decrease $(p=0.0256)$ of Pgc $1 \mathrm{~b}$ expression (Fig. 2g). Furthermore, energy metabolism-related gene expression was detected. Although there was no significant difference of analyzed genes between $\mathrm{PprCl}^{+/}$and WT mice in skeletal muscle (Fig. $2 \mathrm{~m}$ ), expression levels of myosin heavy chain (MHC) isoforms showed a down-regulation of MHC1 (74.6\%, $p<0.05)$ and a 5 -fold up-regulation of MHC IIb $(p<0.05)$ (Fig. 2o), which indicated a less oxidative and more glycolytic alternation in soleus muscle in Pprc1+/- mice. In BAT of $\mathrm{PprCl}^{+/}$mice (Fig. 2i), the compensatory increase of Pgcla expression was followed by significant up-regulation of Cox2, Cox 5b, Gpam and PPAR $\gamma 2$. In WAT the up-regulation of leptin and Ucp1 followed the increased expression of Pgclb. The up-regulation of respiratory chain mRNA and lipid metabolism-related mRNA suggested increased energy expenditure in these tissues.

\section{Deceased liver lipid with expression profiles alterna- tion in Pprc1+/- mice on HFD}

Dissection showed unaltered liver weight in Pprc1+/mice compared with their littermates on HFD (Fig. 3a), whereas histology HE staining showed decreased lipid droplet diameter in Pprc1+/- mice (Fig. 2d). Though no difference was observed in expression analysis of Pgc1 family genes (Fig. 2b), leptin, Gpam, and COX2b showed increased levels in Pprc1+/- mice (Fig. 2c). It suggested an up-regulation of both lipid biogenesis and consumption in Pprc1+/- mice on HFD, which might be an adaptation to the elevation of serum TG (Fig. S1e) and the reduction of hepatic TG (Fig. 3e).

\section{Discussion}

The PGC1 family members are dynamically regulated in response to various signaling pathways involved in nutrient sensing and energy balance. PGCla plays a key role as regulator of adaptive thermogenesis through UCP1 [22], and interacts with CREB to regulate gluconeogenesis [23]. Meanwhile, PGC1b co-activates SREBPs to stimulate lipogenic gene expression [1], and contributes to insulin sensitivity in liver [20, 24]. Regulated by AMPK and SIRT1, PGC1a bridges mitochondrial biogenesis to metabolic signaling pathways [25]. Targeted disruption of either Pgcla $[5,6,26]$ or Pgc $1 b[8,9]$ in mice protects from obesity on both ND and HFD. Due to the fetal lethality of $\mathrm{PprCl}^{-/-}$mice 
Fig. 1a

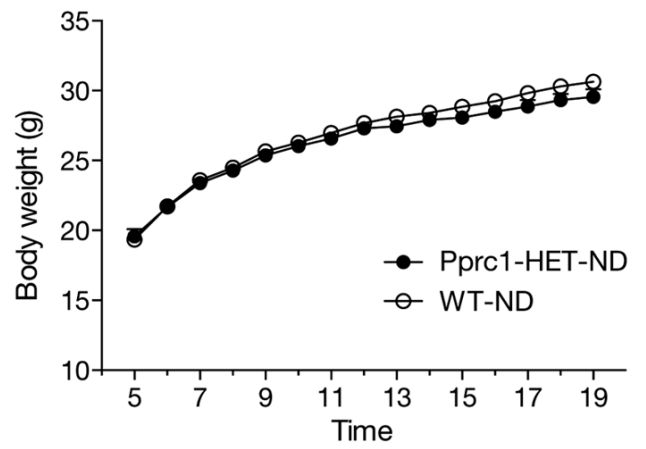

Fig. 1c

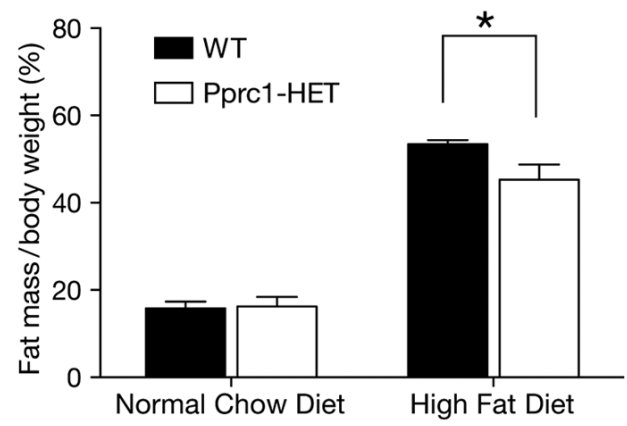

Fig. 1e-1 Pprc1-HET-HFD

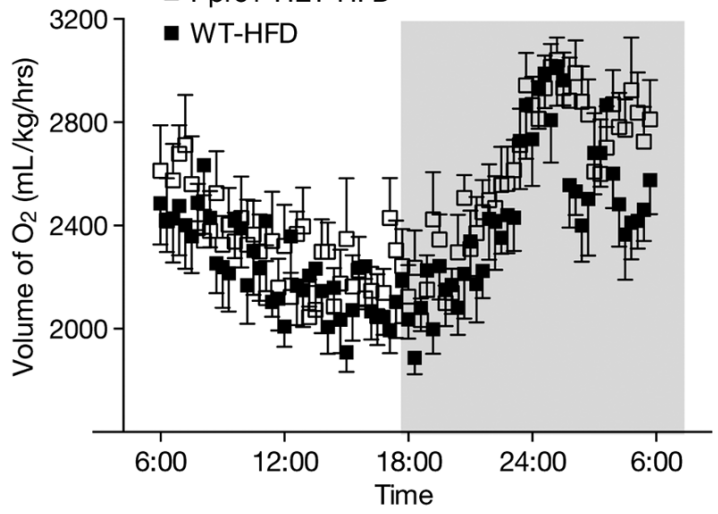

Fig. $1 \mathrm{f}-1$ व Pprc1-HET-HFD

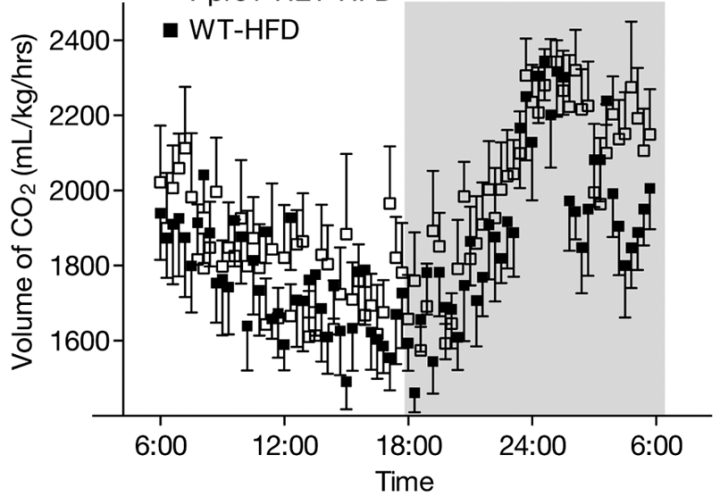

Fig. 1b

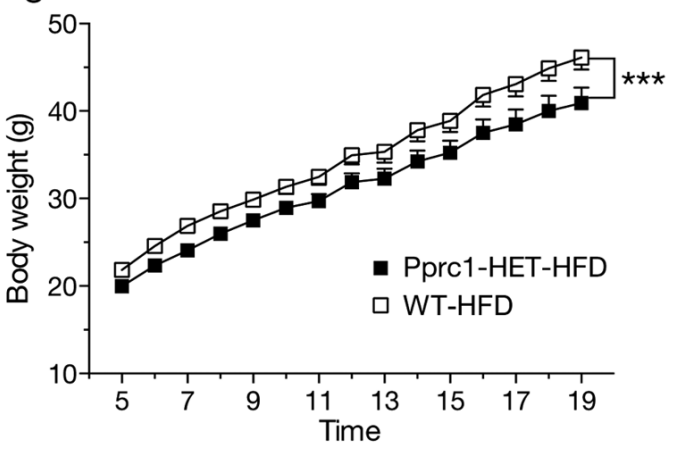

Fig. 1d

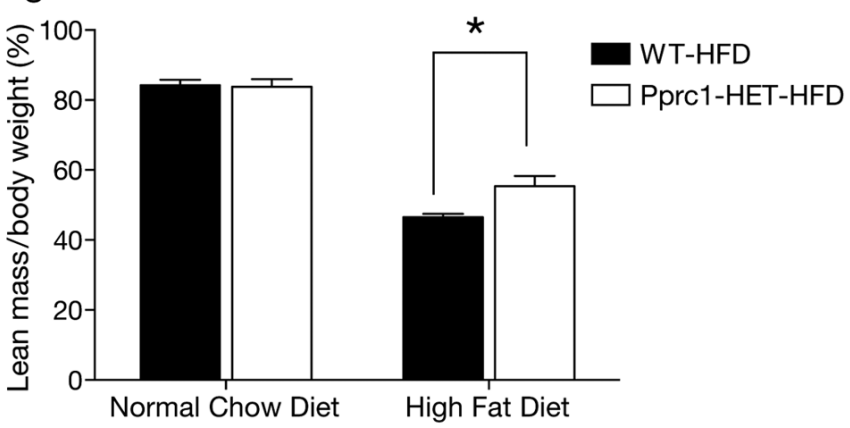

Fig. 1e-2

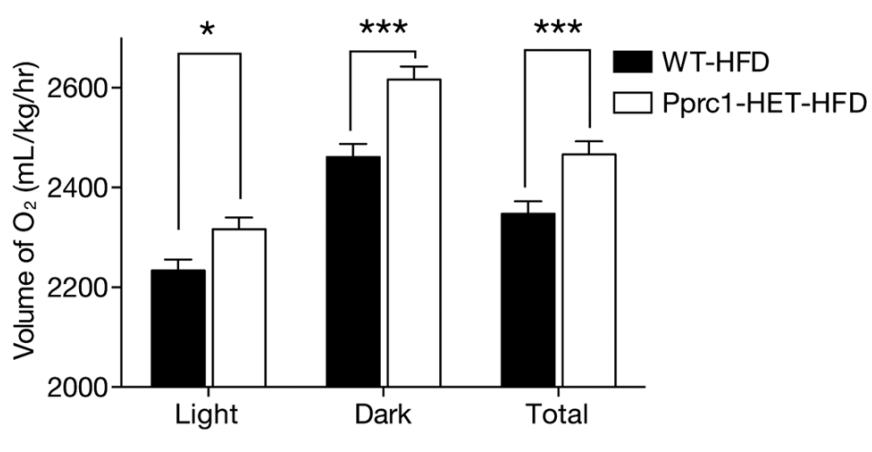

Fig. 1f-2

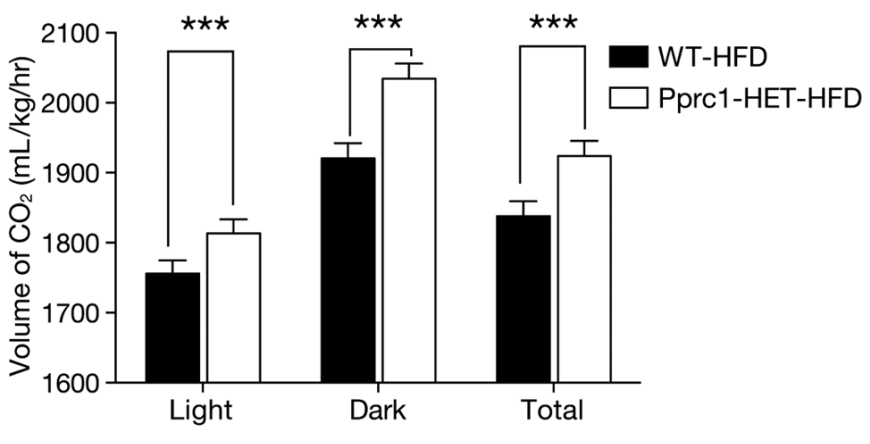


Fig. 1g-1

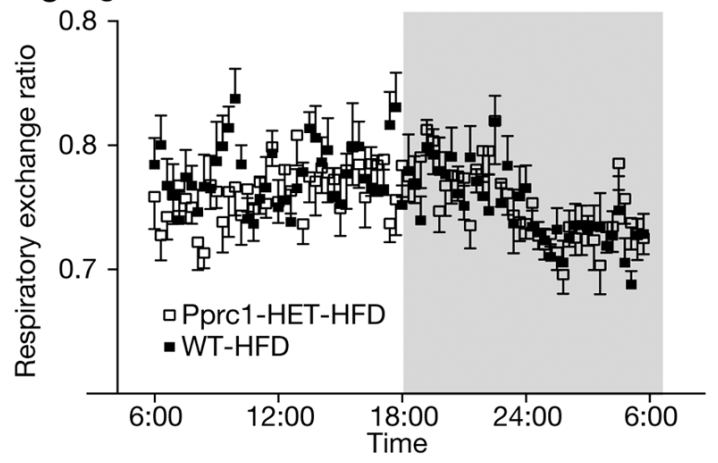

Fig.1h-1

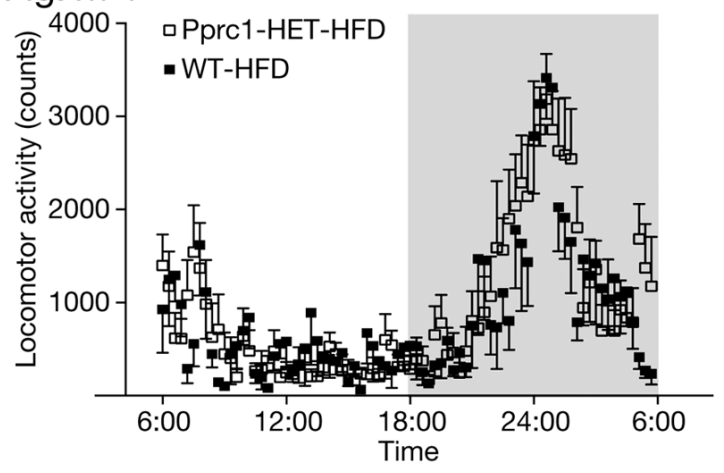

Fig. $1 \mathrm{i}-1$

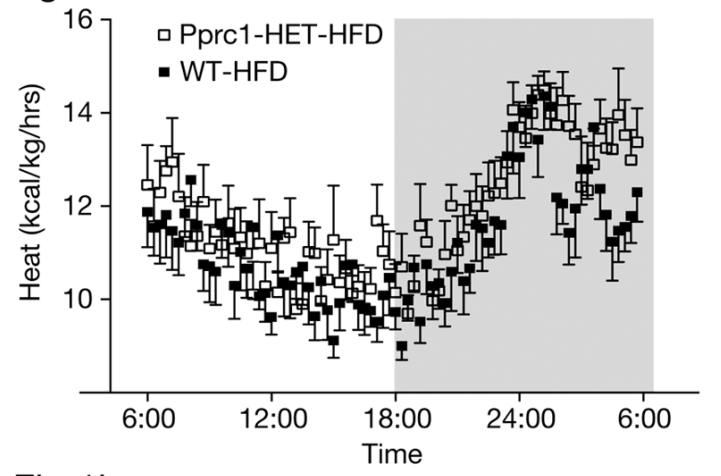

Fig. $1 \mathrm{j}$

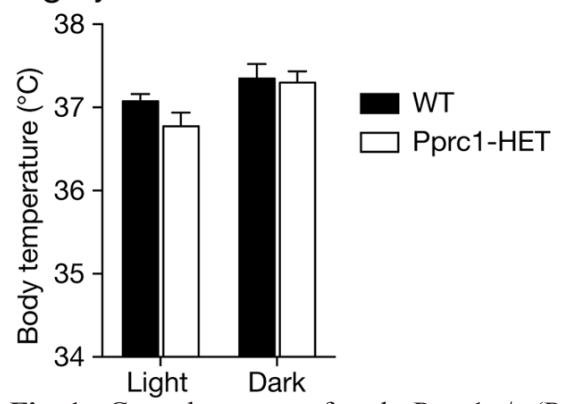

Fig.1g-2

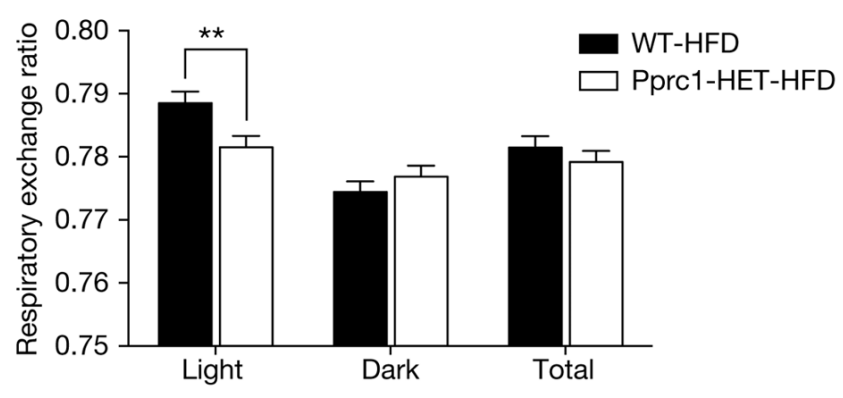

Fig.1h-2

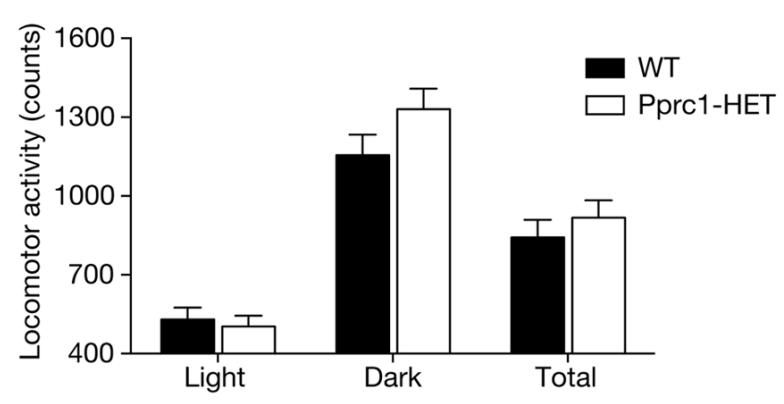

Fig.1i-2 WT-HFD

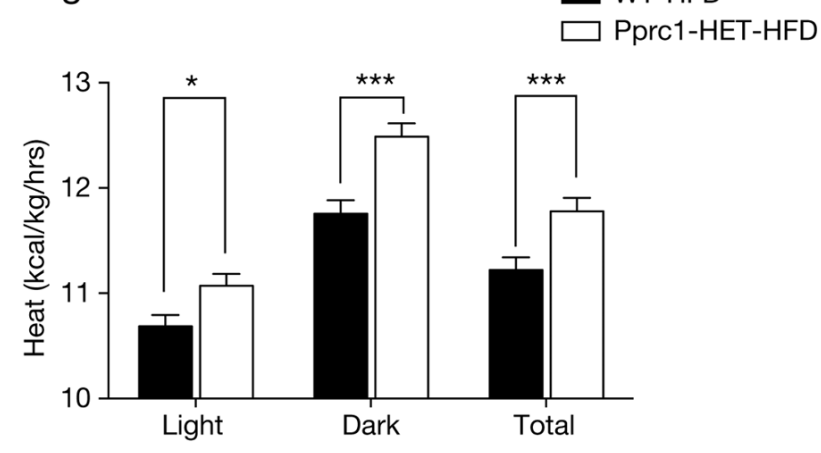

Fig.1k

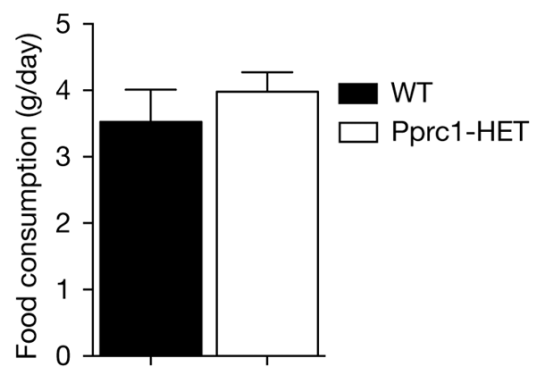

Fig. 1 Growth curves of male Pprc1+/- (Pprc1-HET) mice and wild-type littermates (WT) on ND (a) ( $\mathrm{n}=13-20)$ and HFD (b) $(\mathrm{n}=12-17)$ are shown. Fat (c) and lean (d) mass measured by NMR in 20-week-old mice both on ND and HFD $(\mathrm{n}=8) \mathrm{O}_{2}$ consumption (e), $\mathrm{CO}_{2}$ production (f), RER (g), and heat (i) of Pprc1+/- mice and wild-type littermates on HFD at 20 weeks old were recorded and calculated during the final $24 \mathrm{~h}$ of the procedure. Body temperature $(\mathrm{j})(\mathrm{n}=8)$ and food consumption $(\mathrm{k})(\mathrm{n}$ = 4-8) of mice on HFD were calculated individually for four consecutive days. Bar charts represent the average of each group $(\mathrm{n}=8)$. Error bars show S.E.M. ${ }^{*} p<0.05,{ }^{* *} p<0.01,{ }^{* * *} p<0.001$; WT $v s$. Pprc1-HET mice; Student's $t$ test (c-k) or one-way repeated-measures ANOVA (a-b). 
Fig. $2 \mathrm{a}$

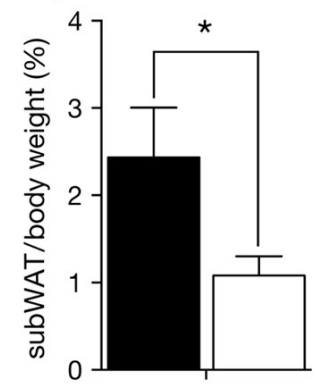

Fig. 2c Wild type

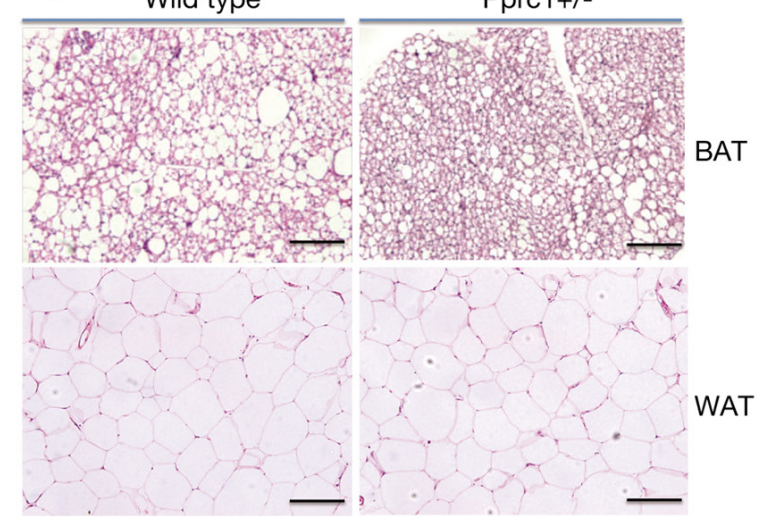

Fig. $2 \mathrm{e}$ White adipose tissue

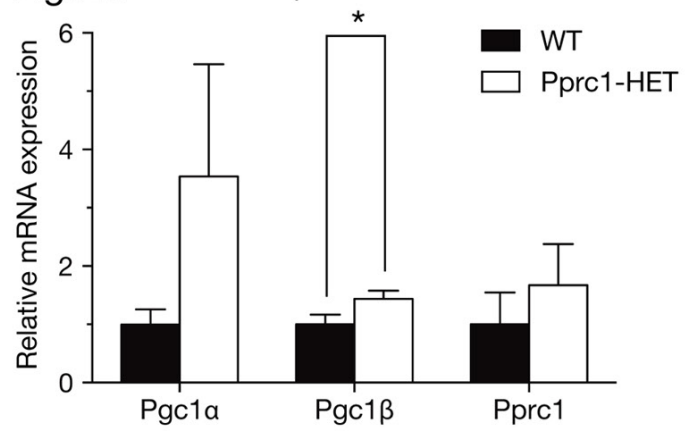

$\begin{array}{lll}\text { Fig. } 2 g & \text { Skeletal muscle } \\ \text { WT } & \square \text { Pprc1-HET }\end{array}$

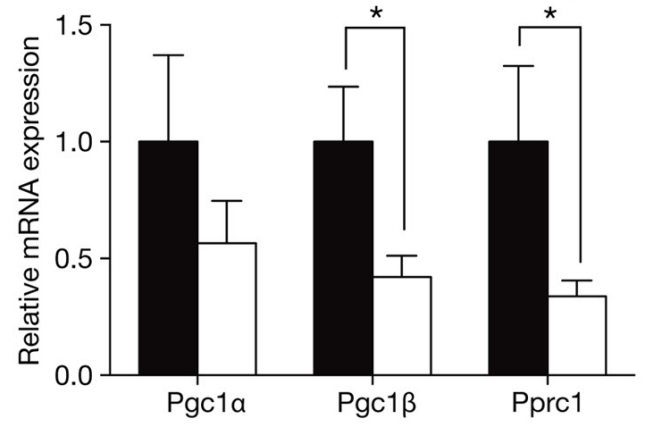

Fig. 2b

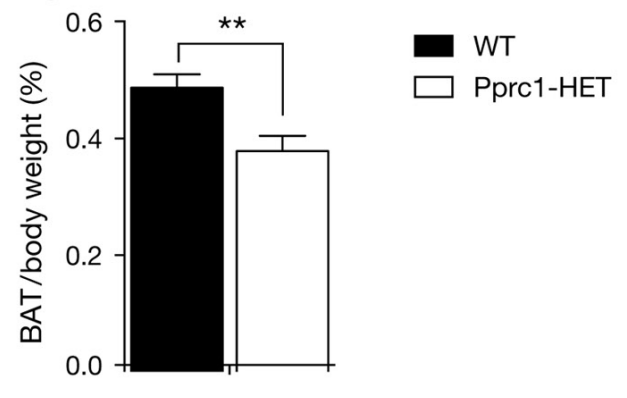

Fig. 2d

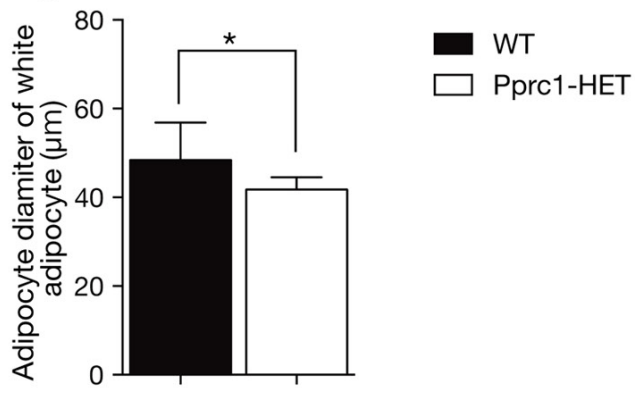

Fig. $2 f \quad$ Brown adipose tissue

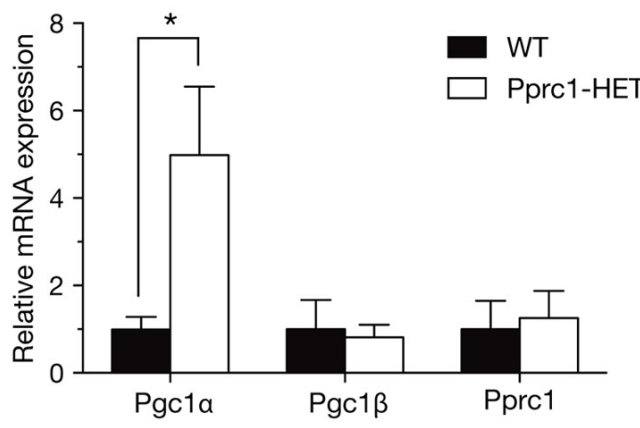

Fig. $2 \mathrm{~h}$ WT Pprc1 $^{+/-}$

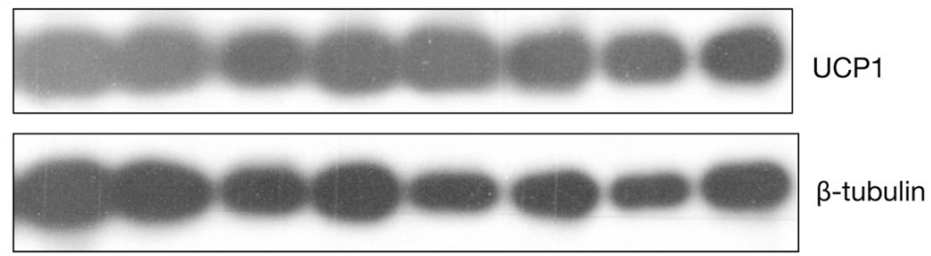




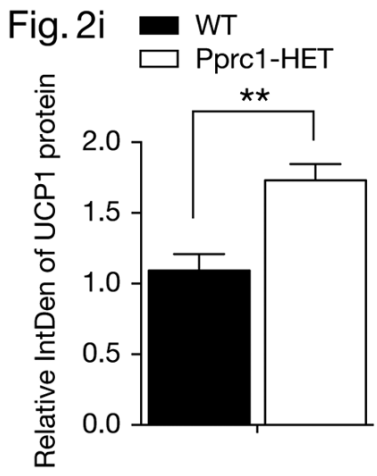

Fig. $2 \mathrm{j}$

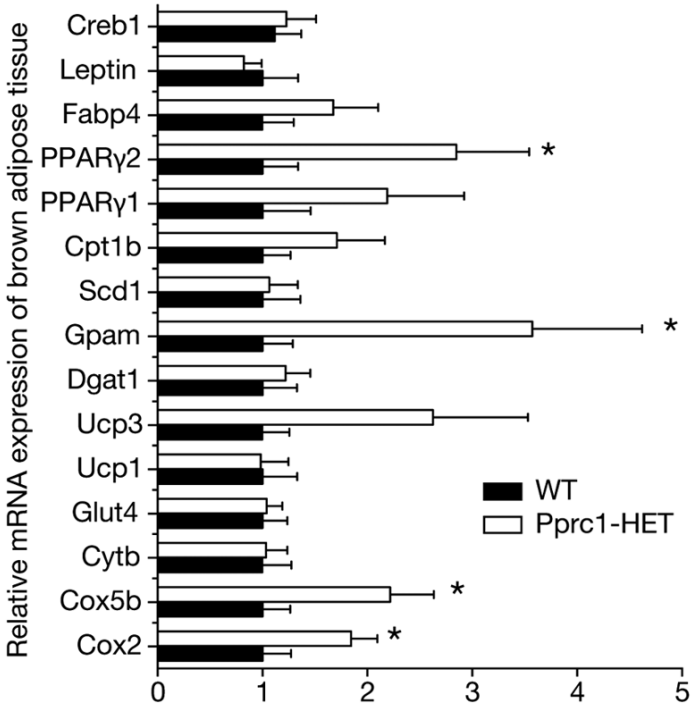

Fig. $2 n$

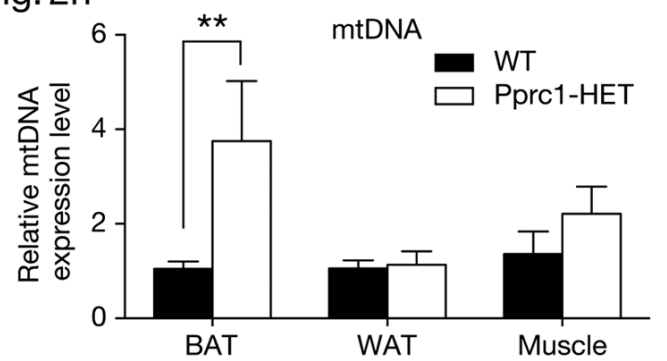

Fig. $2 \mathrm{k}$

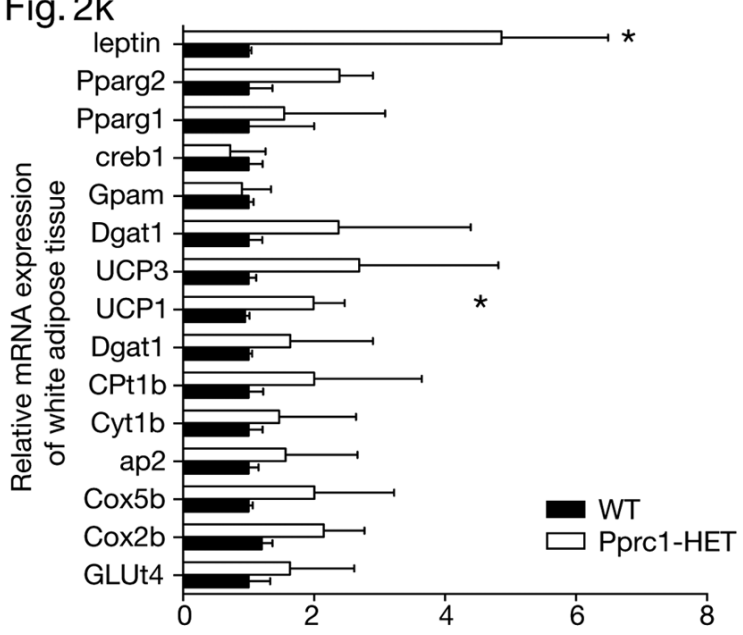

Fig. $2 m$

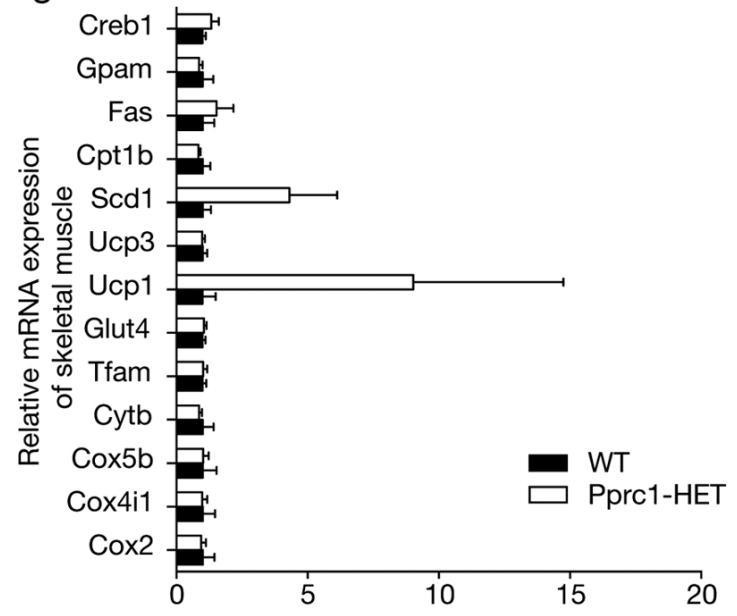

Fig. 20

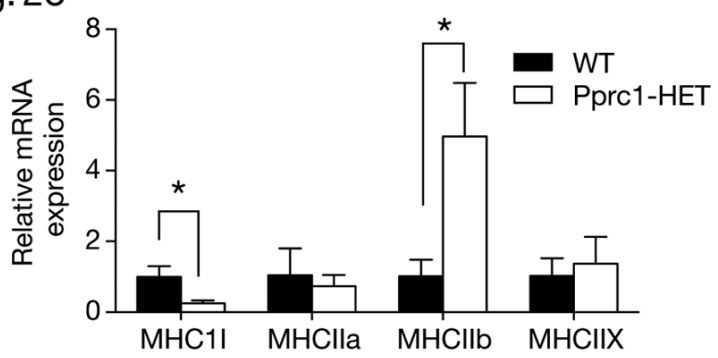

Fig. 2 Comparison of tissue weights of subcutaneous white adipose tissue WAT in lower limb segments (subWAT) (a), interscapular brown adipose tissue (BAT) (b) between 22-week-old male Pprcl $^{+/}$mice and wild-type littermates on HFD ( $\mathrm{n}=8-9$ ). Representative histological images of subWAT and BAT stained with HE in pprcl $^{+/}$mice and wild-type littermates (WT) on HFD (c), Scale bars, $50 \mu \mathrm{m}$. Average lipid-droplet diameter was measured in WAT (d) (n=4-5). Relative mRNA levels of Pgc1 family genes in WAT (e), BAT (f), and soleus muscle (g). Relative mRNA levels of energy metabolism related gene in BAT (j), WAT $(\mathrm{k})$ and soleus muscle $(\mathrm{m})$, relative abundance of mitochondrial DNA in BAT, WAT, and soleus muscle (n). Individual measurements were standardized using GADPH (j-m) or D-loop (n), and then the average of the WT group was set to $1(\mathrm{n}=$ 5-8). Relative mRNA levels of myosin heavy chain (MHC) isoforms (o) in soleus muscle, adjusted GADPH, and then the average of the WT group was set to $1(\mathrm{n}=4-5)$. Western blotting of UCP1 protein levels in BAT (h) in Pprc ${ }^{+/}$mice and wild-type littermates (WT) on HFD $(n=4)$. The right panel represents relative quantification of UCP1 protein (i), normalized to $\beta$-tubulin, and then the average of the WT group was set to 1. Error bars show S.E.M. ${ }^{*} \mathrm{p}<0.05,{ }^{* *} \mathrm{p}<0.01$; WT $v$. Pprc1-HET mice; Student's $t$ test. 

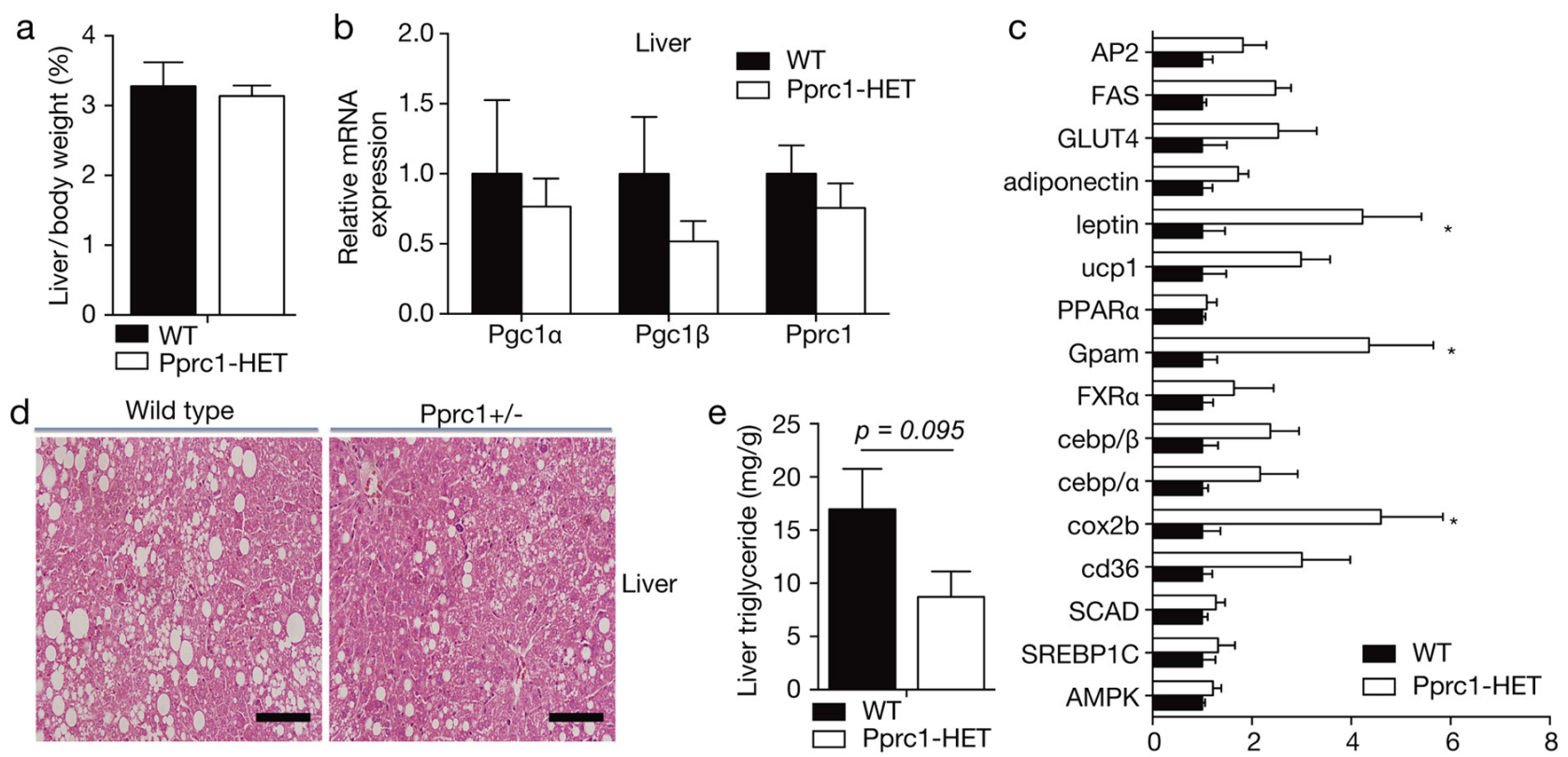

Fig. 3 Comparison of tissue weights of liver (a) between 22-week-old male Pprc1+/- mice and WT littermates on HFD (n = 8-9). Relative mRNA levels of Pgc1 family genes (b) and energy metabolism related gene (c) in liver (b). Individual measurements were standardized using GADPH, and then the average of the WT group was set to $1(n=5-8)$ Representative histological images of liver stained with HE in Pprc1+/- mice and WT littermates on HFD (d), Scale bars, $50 \mu \mathrm{m}$. Hepatic triglyceride (d) on HFD $(\mathrm{n}=8-9)$. Error bars show S.E.M. ${ }^{*} p<0.05,{ }^{* *} p<0.01$; WT $v s$. Pprc1-HET mice; Student’s $t$ test.

[18], the present study is able to investigate the metabolic alteration of $\mathrm{PprCl}^{+/-}$mice, and find that $\mathrm{Pprcl}^{+/-}$ mice are resistant to diet-induced obesity, which is the result of an elevated energy expenditure.

Different from those on ND, male Pprcl $^{+/}$mice on HFD display decrease of body weight and fat mass, along with increase of relative lean mass and heat consumption (Fig. 1). With unaltered locomotor activity, adipocyte tissue was treated as the main organ for the leaner phenotype of $\mathrm{Pprcl}^{+/}$mice. Decrease in relative weights of fat deposits and alternation of adipocyte lipid droplets in both WAT and BAT confirm the remarkable alterations occurring in adipose tissue (Fig. 2). Although the biological and physiological functions of Pprc1 remain largely unknown, there is evidence to suggest the potential role of this gene in metabolic processes. Induced by serum stimulation, Pprcl is an immediate early gene that is rapidly induced in proliferative cell growth $[27,28]$. Pprc1 expression consistently peaks during the first day of embryoid body formation, which suggests that Pprc1 may support the high rate of mitochondrial transcription taking place during early embryogenesis [18]. A microarray screening further revealed that Pprc1 responds to multiple metabolic insults and induces a series of genes involved in inflam- mation, cell stress, and proliferation [29].

It is reported that Pgcla and Pgclb coordinates the formation of slow-twitch muscle fibers [30,31], whereas only in skeletal muscle (soleus) of $\mathrm{Pprcl}^{+/-}$mice, Pprc1 mRNA level shows haplo-insufficiency, with down-regulation of Pgcla and Pgclb, other than in WAT and BAT (Fig. 2). Energy needs in muscle are quite high as living organisms convert chemical energy into mechanical work with the help of muscle [4]. As different muscle fiber types (Type I, Type IIa, Type IIb, and Type II $x$ ) can be distinguished by the type of myosin heavy chain (MHC) isoforms [32-34], increased MHC2b and decreased MHCI in $\mathrm{PprCl}^{+/}$suggests an alternation of fiber type from "slow twitch oxidative" to a more "fast twitch glycolytic" [35]. As high level expression of MHC1 in skeletal muscle is related with low-energy expenditure, the decrease of $\mathrm{MHC} 1$ and increase of MHC2b (Fig. 2o) in skeletal muscle comprise a high energy consumption in $\mathrm{Pprcl}^{+/-}$mice. Though mitochondrial volume remains unaltered, some expression of genes of oxidative phosphorylation increased. Consistent with the phenotype of PGC1a-ko mice [36], it suggests that Pprc1, as well as other Pgc1s, could participate in the energy consumption process in a tissue-specific manner. On the other hand, consistent with a previ- 
ously reported up-regulation of Pgcla in the absence of Pgc1b $[8,9]$, our results further show the compensatory increase of other Pgc1 family member that could induce an up-regulation of respiratory chain mRNA in $\mathrm{PprCl}^{+/-}$ mice, suggesting a distinctively co-operative and compensatory relationship prevailing among the three $\mathrm{Pgc1}$ family members in physiological condition.

Interestingly, this study provides yet another example of increasing serum TG level in leaner mice, in addition to the one previously reported [37]. The increase of serum TG in $\mathrm{Pprcl}^{+/}$mice is consistent with the up-regulation of Gpam expression in BAT and in liver (Fig. 2i, 3c). Gpam is supposed to initiate hepatic de novo $\mathrm{TG}$ synthesis both in vitro $[38,39,26]$ and in vivo [40]. Liver-directed overexpression of Gpam in mice results in increased plasma TAG [41]. However, histological examination of liver tissue from $\mathrm{PprCl}^{+/-}$mice revealed a reduction in lipid droplets compared with their littermates (Fig. 3d). Consistent with this histology, a tendency of reduction in hepatic triglycerides was seen (Fig. 3e), as well as an increase in hepatic expression of fatty acid oxidation genes (Fig. 3c). This suggests the possibility that up-regulated hepatic fatty acid oxidation and increased hepatic de novo TG syn- thesis happened simultaneously in $\mathrm{Pprcl}^{+/}$mice on HFD, and that oxidation may have exceeded synthesis.

Taken together, these observations demonstrate an elevated basal metabolic rate and lipid metabolic alteration of $\mathrm{PprCl}^{+/-}$mice on HFD, and indicate a significant role of Pprc1 in controlling mitochondrial gene expression and energy metabolic process, synergistically with Pgcla and Pgc1b.

\section{Acknowledgments}

This work was supported in part by grants from the Key Laboratory for Endocrine and Metabolic Diseases of the Ministry of Public Health (No. 1994DP131044); the National Natural Science Foundation of China (No. 81070670); the National Natural Science Foundation of China (No. 81100588); and the National Natural Science Foundation of China (No. 81370934).

\section{Disclosure}

None of the authors have any potential conflicts of interest associated with this research.

Supplementary Table 1 Sequences for real-time PCR primers

\begin{tabular}{|c|c|c|}
\hline Target & Forward primer $\left(5^{\prime}->3^{\prime}\right)$ & Reverse primer (5'->3') \\
\hline Gapdh & TGACCACAGTCCATGCCATC & GACGGACACATTGGGGGTAG \\
\hline $\operatorname{Pgc} 1 \alpha$ & TATGGAGTGACATAGAGTGTGCT & CCACTTCAATCCACCCAGAAAG \\
\hline $\operatorname{Pgc} 1 \beta$ & TCCTGTAAAAGCCCGGAGTAT & GCTCTGGTAGGGGCAGTGA \\
\hline Pprc1 & CAGGAGAAGAAGCCCTTAGACC & CTTTCGCCAAGAGTGAGACAG \\
\hline Cox2 & GCTCCTTAGTCCTCTATATCAT & TGCGTCTATTGTGCTTGTA \\
\hline Cox4i & CCATGTCACGATGCTGTCTG & GATGCGGTACAACTGAACTTTCT \\
\hline Cox $5 b$ & GGAAGACCCTAATCTAGTCCCG & GTTGGGGCATCGCTGACTC \\
\hline Tfam & ATTCCGAAGTGTTTTTCCAGCA & TCTGAAAGTTTTGCATCTGGGT \\
\hline Cytb & GGACGAGGCTTATATTATGGA & AATGCTGTGGCTATGACTG \\
\hline Glut4 & GTGACTGGAACACTGGTCCTA & CCAGCCACGTTGCATTGTAG \\
\hline Ucp1 & AGGCTTCCAGTACCATTAGGT & CTGAGTGAGGCAAAGCTGATTT \\
\hline Ucp3 & CTGCACCGCCAGATGAGTTT & ATCATGGCTTGAAATCGGACC \\
\hline Cpt1b & AGGCACTTCTCAGCATGGTC & GCTTCAGGGTTTGTCGGAAGA \\
\hline Dgat1 & GTGCCATCGTCTGCAAGATTC & GCATCACCACACACCAATTCAG \\
\hline Fas & GCGGGTTCGTGAAACTGATAA & GCAAAATGGGCCTCCTTGATA \\
\hline Gpam & TCTCTGGGTTTGCGGAATGTT & GCCTTGTGTGCGTTTCATTGAT \\
\hline Scd1 & TTCTTGCGATACACTCTGGTGC & CGGGATTGAATGTTCTTGTCGT \\
\hline PPAR $\gamma 1$ & GTCACACTCTGACAGGAGCC & AGAACGTGACTTCTCAGCCC \\
\hline PPAR $\gamma 2$ & TCTCCTGTTGACCCAGAGCAT & CCACAGAGCTGATTCCGAAGT \\
\hline Creb1 & AGCAGCTCATGCAACATCATC & AGTCCTTACAGGAAGACTGAACT \\
\hline Fabp4 & AAGGTGAAGAGCATCATAACCCT & TCACGCCTTTCATAACACATTCC \\
\hline Leptin & GTTAGACCCTACTCACGGCG & TGTGCCCTGAAATGCGGTAT \\
\hline MHC I & CCAAGGGCCTGAATGAGGAG & GCA AAG GCT CCA GGT CTG AG \\
\hline MHC II a & AAG CGA AGA GTA AGG CTG TC & GTG ATT GCT TGC AAA GGA AC \\
\hline MHC II b & CGA AGG CGG AGC TAC GGT CA & CGG CAG CCA CTT GTA GGG GT \\
\hline MHC II x & GCC AGG GTC CGT GAA CTT GAA G & CCT CCG CTT CCT CAG CTT GTC T \\
\hline $18 \mathrm{~S}$ & ACCGCAGCTAGGAATAATGGA & ACCTAGTTCCGAAAACCA \\
\hline
\end{tabular}



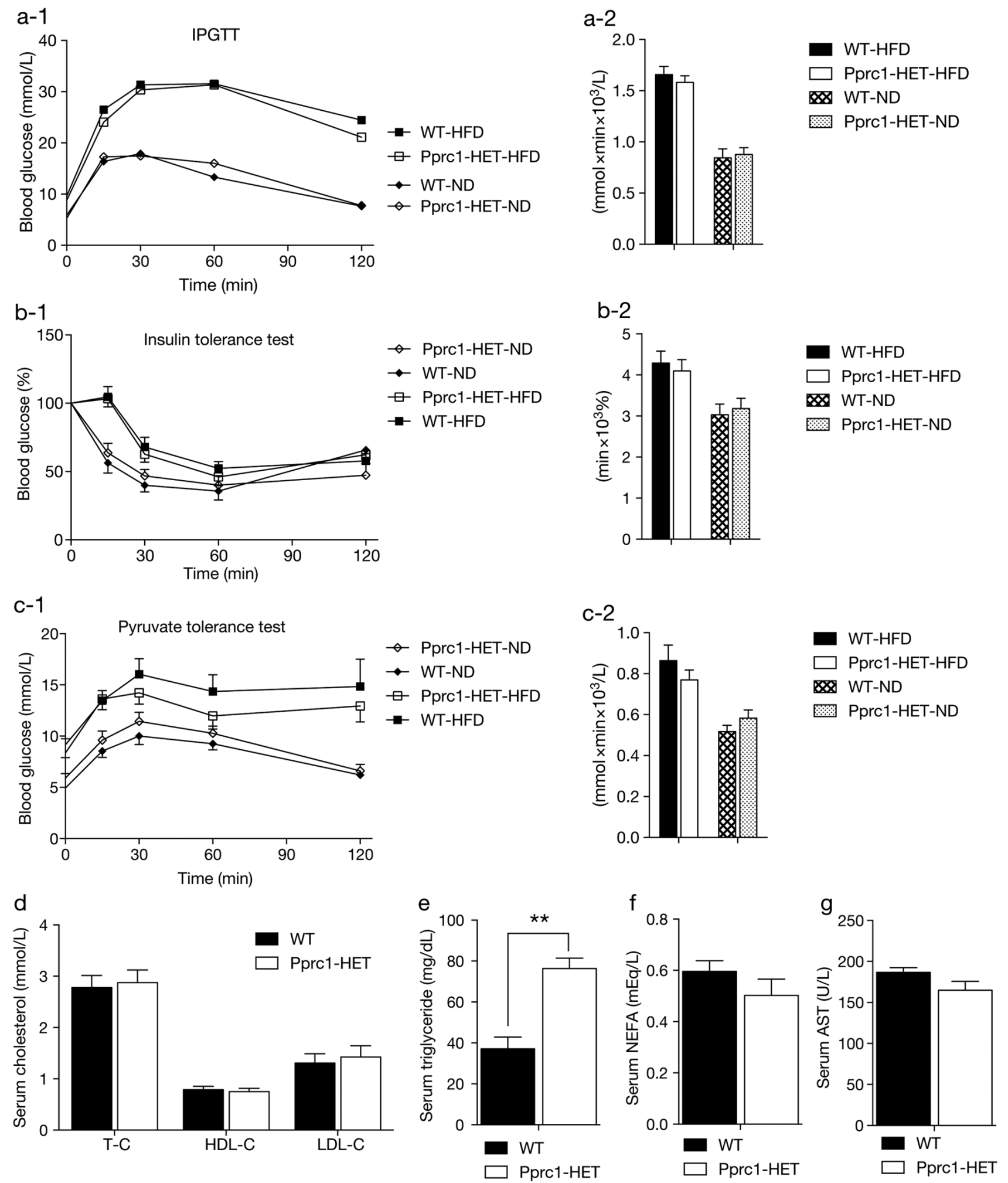

Supplementary Fig. S1 Intra-peritoneal glucose tolerance test (IPGTT) (a), intra-peritoneal insulin tolerance test (ITT) (b) and intra-peritoneal pyruvate tolerance test (PTT) (c) of Pprc1+/- mice and wild-type littermates on ND and HFD at 21 weeks old. The bar charts represent the average area under the curve (AUC) $(n=7-8)$. Serum cholesterol (d), triglyceride (TG) (e), non-esterified fatty acid (NEFA) (f), and aspartate transaminase (AST) (g) of 22-week-old male Pprc1+/- mice and wild-type littermates on HFD $(\mathrm{n}=8-9)$. Error bars show S.E.M. $* * p<0.01$; WT $v s$. Pprc1-HET mice. 


\section{References}

1. Lin J, Yang R, Tarr PT, Wu PH, Handschin C, et al. (2005) Hyperlipidemic effects of dietary saturated fats mediated through PGC-1beta coactivation of SREBP. Cell 120: 261-273.

2. Lin J, Handschin C, Spiegelman BM (2005) Metabolic control through the PGC-1 family of transcription coactivators. Cell Metab 1: 361-370.

3. Finck BN, Kelly DP (2006) PGC-1 coactivators: inducible regulators of energy metabolism in health and disease. J Clin Invest 116: 615-622.

4. Andersen G, Wegner L, Yanagisawa K, Rose CS, Lin J, et al. (2005) Evidence of an association between genetic variation of the coactivator PGC-1beta and obesity. $J$ Med Genet 42: 402-407.

5. Lin J, Wu PH, Tarr PT, Lindenberg KS, St-Pierre J, et al. (2004) Defects in adaptive energy metabolism with CNS-linked hyperactivity in PGC-1alpha null mice. Cell 119: 121-135.

6. Leone TC, Lehman JJ, Finck BN, Schaeffer PJ, Wende AR, et al. (2005) PGC-1alpha deficiency causes multisystem energy metabolic derangements: muscle dysfunction, abnormal weight control and hepatic steatosis. PLoS Biol 3: e101.

7. Lehman JJ, Barger PM, Kovacs A, Saffitz JE, Medeiros DM, et al. (2000) Peroxisome proliferator-activated receptor gamma coactivator-1 promotes cardiac mitochondrial biogenesis. J Clin Invest 106: 847-856.

8. Sonoda J, Mehl IR, Chong LW, Nofsinger RR, Evans RM (2007) PGC-1beta controls mitochondrial metabolism to modulate circadian activity, adaptive thermogenesis, and hepatic steatosis. Proc Natl Acad Sci U S A 104: 5223-5228.

9. Lelliott CJ, Medina-Gomez G, Petrovic N, Kis A, Feldmann HM, et al. (2007) Ablation of PGC-1beta results in defective mitochondrial activity, thermogenesis, hepatic function, and cardiac performance. PLoS Biol 4: e369.

10. Kamei Y, Ohizumi H, Fujitani Y, Nemoto T, Tanaka T, et al. (2003) PPARgamma coactivator 1beta/ERR ligand 1 is an ERR protein ligand, whose expression induces a high-energy expenditure and antagonizes obesity. Proc Natl Acad Sci U S A 100: 12378-12383.

11. Edwards TL, Velez Edwards DR, Villegas R, Cohen SS, Buchowski MS, et al. (2012) HTR1B, ADIPOR1, PPARGC1A, and CYP19A1 and obesity in a cohort of Caucasians and African Americans: an evaluation of gene-environment interactions and candidate genes. Am J Epidemiol 175: 11-21.

12. Ridderstrale M, Johansson LE, Rastam L, Lindblad $\mathrm{U}$ (2006) Increased risk of obesity associated with the variant allele of the PPARGC1A Gly482Ser polymorphism in physically inactive elderly men. Diabetologia 49: 496-500.
13. Park KS, Shin HD, Park BL, Cheong HS, Cho YM, et al. (2006) Putative association of peroxisome proliferator-activated receptor gamma co-activator 1 beta (PPARGC1B) polymorphism with Type 2 diabetes mellitus. Diabet Med 23: 635-642.

14. Goyenechea E, Crujeiras AB, Abete I, Parra D, Martinez JA (2008) Enhanced short-term improvement of insulin response to a low-caloric diet in obese carriers the Gly482Ser variant of the PGC-1alpha gene. Diabetes Res Clin Pract 82: 190-196.

15. Gemma C, Sookoian S, Alvarinas J, Garcia SI, Quintana L, et al. (2009) Maternal pregestational BMI is associated with methylation of the PPARGC1A promoter in newborns. Obesity (Silver Spring) 17: 1032-1039.

16. Geloneze SR, Geloneze B, Morari J, Matos-Souza JR, Lima MM, et al. (2012) PGC1alpha gene Gly482Ser polymorphism predicts improved metabolic, inflammatory and vascular outcomes following bariatric surgery. Int J Obes (Lond) 36: 363-368.

17. Reiling E, van Vliet-Ostaptchouk JV, van 't Riet E, van Haeften TW, Arp PA, et al. (2009) Genetic association analysis of 13 nuclear-encoded mitochondrial candidate genes with type II diabetes mellitus: the DAMAGE study. Eur J Hum Genet 17: 1056-1062.

18. He X, Sun C, Wang F, Shan A, Guo T, et al. (2012) Peri-implantation lethality in mice lacking the PGC-1related coactivator protein. Dev Dyn 241: 975-983.

19. Bohlooly-Y M, Olsson B, Bruder CE, Lindén D, Sjögren $\mathrm{K}$, et al. (2005) Growth hormone overexpression in the central nervous system results in hyperphagia-induced obesity associated with insulin resistance and dyslipidemia. Diabetes 54: 51-62.

20. Nagai Y, Yonemitsu S, Erion DM, Iwasaki T, Stark R, et al. (2009) The role of peroxisome proliferator-activated receptor gamma coactivator-1 beta in the pathogenesis of fructose-induced insulin resistance. Cell Metab 9: 252-264.

21. Philp A, Belew MY, Evans A, Pham D, Sivia I, et al. (2011) The PGC-1alpha-related coactivator promotes mitochondrial and myogenic adaptations in $\mathrm{C} 2 \mathrm{C} 12$ myotubes. Am J Physiol Regul Integr Comp Physiol 301: R864-872.

22. Wu Z, Puigserver P, Andersson U, Zhang C, Adelmant G, et al. (1999) Mechanisms controlling mitochondrial biogenesis and respiration through the thermogenic coactivator PGC-1. Cell 98: 115-124.

23. Herzig S, Long F, Jhala US, Hedrick S, Quinn R, et al. (2001) CREB regulates hepatic gluconeogenesis through the coactivator PGC-1. Nature 413: 179-183.

24. Vianna CR, Huntgeburth M, Coppari R, Choi CS, Lin J, et al. (2006) Hypomorphic mutation of PGC-1beta causes mitochondrial dysfunction and liver insulin resistance. Cell Metab 4: 453-464. 
25. Scarpulla RC, Vega RB, Kelly DP (2012) Transcriptional integration of mitochondrial biogenesis. Trends Endocrinol Metab 23: 459-466.

26. Linden D, William-Olsson L, Rhedin M, Asztely AK, Clapham JC, et al. (2004) Overexpression of mitochondrial GPAT in rat hepatocytes leads to decreased fatty acid oxidation and increased glycerolipid biosynthesis. J Lipid Res 45: 1279-1288.

27. Andersson U, Scarpulla RC (2001) Pgc-1-related coactivator, a novel, serum-inducible coactivator of nuclear respiratory factor 1-dependent transcription in mammalian cells. Mol Cell Biol 21: 3738-3749.

28. Vercauteren K, Pasko RA, Gleyzer N, Marino VM, Scarpulla RC (2006) PGC-1-related coactivator: immediate early expression and characterization of a CREB/ NRF-1 binding domain associated with cytochrome $\mathrm{c}$ promoter occupancy and respiratory growth. Mol Cell Biol 26: 7409-7419.

29. Gleyzer N, Scarpulla RC (2011) PGC-1-related coactivator (PRC), a sensor of metabolic stress, orchestrates a redox-sensitive program of inflammatory gene expression. J Biol Chem 286: 39715-39725.

30. Arany Z, Lebrasseur N, Morris C, Smith E, Yang W, et al. (2007) The transcriptional coactivator PGC-1beta drives the formation of oxidative type IIX fibers in skeletal muscle. Cell Metab 5: 35-46.

31. Lin J, Wu H, Tarr PT, Zhang CY, Wu Z, et al. (2002) Transcriptional co-activator PGC-1 alpha drives the formation of slow-twitch muscle fibres. Nature 418: 797801.

32. Schiaffino S, Reggiani C (2011) Fiber types in mammalian skeletal muscles. Physiol Rev 91: 1447-1531.

33. Bottinelli R (2001) Functional heterogeneity of mammalian single muscle fibres: do myosin isoforms tell the whole story? Pflugers Arch 443: 6-17.

34. van Wessel T, de Haan A, van der Laarse WJ, Jaspers RT
(2010) The muscle fiber type-fiber size paradox: hypertrophy or oxidative metabolism? Eur J Appl Physiol 110: 665-694.

35. Pette D, Staron RS (2000) Myosin isoforms, muscle fiber types, and transitions. Microsc Res Tech 50: 500509.

36. Arany Z, He H, Lin J, Hoyer K, Handschin C, et al. (2005) Transcriptional coactivator PGC-1 alpha controls the energy state and contractile function of cardiac muscle. Cell Metab 1: 259-271.

37. Setsuie R, Suzuki M, Kabuta T, Fujita H, Miura S, et al. (2009) Ubiquitin C-terminal hydrolase-L3-knockout mice are resistant to diet-induced obesity and show increased activation of AMP-activated protein kinase in skeletal muscle. FASEB J 23: 4148-4157.

38. Igal RA, Wang S, Gonzalez-Baro M, Coleman RA (2001) Mitochondrial glycerol phosphate acyltransferase directs the incorporation of exogenous fatty acids into triacylglycerol. J Biol Chem 276: 42205-42212.

39. Adjeitey CN, Mailloux RJ, Dekemp RA, Harper ME (2013) Mitochondrial uncoupling in skeletal muscle by UCP1 augments energy expenditure and glutathione content while mitigating ROS production. Am J Physiol Endocrinol Metab 305: E405-415.

40. Hammond LE, Gallagher PA, Wang S, Hiller S, Kluckman KD, et al. (2002) Mitochondrial glycerol-3-phosphate acyltransferase-deficient mice have reduced weight and liver triacylglycerol content and altered glycerolipid fatty acid composition. Mol Cell Biol 22: 8204-8214.

41. Linden D, William-Olsson L, Ahnmark A, Ekroos K, Hallberg C, et al. (2006) Liver-directed overexpression of mitochondrial glycerol-3-phosphate acyltransferase results in hepatic steatosis, increased triacylglycerol secretion and reduced fatty acid oxidation. FASEB J 20: 434-443. 